\title{
Light and Electron Microscopic Observations of Mitochondrial Fusion in Plasmodia Induced Sporulation in Physarum polycephalum
}

\author{
Soryu Nishibayashi \\ Plantech Research Institute, Mitsubishi Chemical Ind. Research Center, \\ 1000 Kamoshida-cho, Midori-ku, Yokohama, 227 Japan \\ and \\ Shigeyuki Kawana and Tsuneyoshi Kuroiwa \\ Department of Cell Biology, National Institute for Basic Biology, \\ 38 Nishigonaka, Myodaiji-cho, Okazaki, 444 Japan
}

Accepted June 6, 1986

The growing plasmodium of Physarum polycephalum provides a system for the investigation of mitochondrial division. In Physarum polycephalum mitochondrial division consists of two main processes, mitochondrial kinesis and mitochondrial nuclear division (Kuroiwa et al. 1977). Mitochondrial division is semi-synchronized in the plasmodium (Kuroiwa et al. 1978) Light and thin-sectioned electron microscopic observations have shown the presence of a mitochondrial nucleus (mt-nucleus) situated in the central portion of the inner matrix of $P$. polycepahlum mitochondria throughout the mitochondrial division cycle (Guttes et al. 1967, Kuroiwa 1973, Kuroiwa et al. 1977). The mt-nucleus elongates longitudinally with the growth of mitochondria. Just before mitochondrial division, the mt-nucleus divides by constriction of the limiting membrane of the dividing mitochondria and is distributed almost equally to the daughter mitochondria. The mt-DNA content per mitochondrial nucleus is estimated to be about 32 molecules, which are linear and have molecular weights of $45 \times 10^{6} \mathrm{D}$ per mitochondrion in spherical mitochondria (Kawano et al. 1982). This is contrast to the finding that during spherulation in $P$. polycephalum mitochondria and mitochondrial nuclei become smaller (Kawano et al. 1983). Diminutive mitochondria contain very small particle-shaped mitochondrial nuclei and the amount of DNA, RNA and protein per mitochondrion decreases remarkably with spherulation (Kawano et al. 1983). Thus, in $P$. polycephalum mitochondrial differentiation to inactive state (spherulation) was known to be characterized by a decrease not only in dimension but also in DNA, RNA and protein content.

Very little information was available, however, concerning the behavior of mitochondria and mt-nuclei during sporulation. The present paper described the behavior of cell nuclei, mitochondria and mt-nuclei during sporulation, during which starved plasmodia differentiate into sporangia.

\section{Materials and methods}

\section{Induction of sporulation}

To induce sporulation, plasmodia reactivated from sclerotia were obtained according to the method of Yoshimoto and Kamiya (1978). The stock culture was grown with compressed oats in a plastic bucket lined with sheets of wet filter paper. The outgrowth of the plasmodium was detached, together with the filter paper, from the side wall of the bucket and dried slowly for one day. Sclerotia obtained by this procedure were stocked in a desiccator. To reactivate the sclerotia, a sheet of $1.5 \%$ agar was prepared with tap water on the flat bottom of a shallow 
rectangular plastic container $(20 \times 30 \mathrm{~cm})$ with a cover. A piece of the sclerotium weighing $0.3 \mathrm{~g}$ was then placed on one corner of the agar plate and left standing overnight without feeding. In the course of 16-24 hrs, the sclerotium on the agar plate developed an active plasmodium, and finally differentiated into fruiting bodies. The sclerotia and starved plasmodia were grown at $20^{\circ} \mathrm{C}$ in the dark, but they were sometimes exposed to light so that their morphological changes, cell nuclei, mitochondria and mt-nuclei could be observed.

\section{Induction of amoebae}

To observe mt-nuclei of amoebae by light and electron microscopy, spores were germinated on a non-nutrient agar plate after thorough washing with $1 / 15 \mathrm{M}$ phosphate buffer (pH 6.8). Amoebae appeared within one day after plating.

\section{Observation of sporulation processes}

Morphological changes and time course during sporulation were investigated using a Sony Video Camera with a macro lens and a National Time Lapse VTR NV-8030, at $20^{\circ} \mathrm{C}$ under continuous weak light conditions. The camera image was projected in real time on a television monitor (Ikegami PM-121T) and simultaneously recorded on magnetic tape. Photographs during sporulation were taken at a magnification of $\times 10$ on $35 \mathrm{~mm}$ Fuji Neopan F film (ASA 32).

\section{DAPI staining}

Samples at various stages of sclerotium, plasmodium, sporulation and amoeba were put on glass slides, fixed in a drop of buffer $\mathrm{NS}(20 \mathrm{mM}$ Tris- $\mathrm{HCl}$ at $\mathrm{pH} 7.6,0.25 \mathrm{M}$ sucrose, $1 \mathrm{mM}$ EDTA, $1 \mathrm{mM} \mathrm{MgCl}, 0.1 \mathrm{mM} \mathrm{ZnSO}, 0.1 \mathrm{mM} \mathrm{CaCl}_{2}, 0.8 \mathrm{mM}$ phenylmethyl-sulfonylfluoride, $0.05 \% 2$-mercaptoethanol), dissolved in $1 \%$ glutaraldehyde to stabilize the cell and mitochondrial nuclei, and immediately stained with a drop of buffer NS containing $2 \mu \mathrm{g} / \mathrm{ml}$ 4' 6-diamidino2-phenylindole (DAPI) (Nishibayashi and Kuroiwa 1982). A coverslip was then placed on the mixture and pressed gently onto each sample.

\section{Epifluorescence microscopy}

DAPI fluorescence was excited by light from a $100 \mathrm{w} \mathrm{Hg}$ lamp in an Olympus BHS-RFK epifluorescent microscope. Observations of DAPI fluorescence were done with a UVFL 100/ 1.30 objective, using a UV excitation filter $(365 \mathrm{~nm}$ ) in combination with a $420 \mathrm{~nm}$ suppression filter (Olympus, Tokyo) (Nishibayashi and Kuroiwa 1982). Photographas were taken at a magnification of $\times 625$ on $35 \mathrm{~mm}$ Fuji 400 film with a 16-sec exposure time.

\section{Measurement of fluorescence intensity}

The fluorescence intensity emitted by the DAPI-stained cells and mitochondrial nuclei was measured using a Zeiss-MPM03-Fluorometer equipped with a high-pressure mercury lamp XBO $100 \mathrm{w}$, filter combination No. 487711 and a Neofluor 100/1.30 oil objective (Nishibayashi and Kuroiwa 1985). The areas of the individual cells and mt-nuclei were optically isolated by the $8 \mu \mathrm{m}$ MPM diaphragm for cell nuclei and by the $2 \mu \mathrm{m}$ MPM diaphragm for mt-nuclei.

\section{Electron microscopy}

Samples at various stages during sporulation were fixed for $3 \mathrm{hrs}$ in ice-cold $2 \%$ glutaraldehyde buffer with sodium cacodylate to $\mathrm{pH} 7.0$, washed in sodium cacodylate buffer $\mathrm{pH} 7.0$ for $1 \mathrm{hr}$, and postfixed in $2 \% \mathrm{OsO}_{4}$ for $12 \mathrm{hrs}$. They were then dehydrated in a graded series of ethanol and propylene oxide (30 min at each step) and embedded in Spurr's epoxy resin (Spurr 1969). Ultra-thin serial sections were cut with glass knives on a Porter-Bulum microtome and 
the sections were mounted on grids coated with Formvar. Section thickness was about $1000 \AA$. Thin sections were stained with saturated uranyl acetate and lead citrate. The specimens were observed with a Hitachi HU 500 electron microscope at $75 \mathrm{KV}$.

\section{Three-dimensional reconstruction}

All sections of an entire mitochondrion were photographed at magnifications of $\times 12000$ and $\times 20000$. The outlines of mitochondria and mt-nuclei were drawn onto the tracing papercovered electron micrographs of mitochondria and mt-nuclei enlarged up to a magnification of $\times 42000$. The outlines of all mitochondria and mt-nuclei on the tracing papers were fed into a computer by tracing with a cursor on the digitizing table. Three-dimensional reconstruction of each of the mitochondria and mt-nuclei was obtained by computer-aided graphics, and the reconstructed profiles were projected on a terminal display and printed out on a plotter (Nikon Cosmozone System 2).

\section{Observations and results}

\section{Morphological changes during sporulation}

The starved plasmodia which reactivated from sclerotia on a non-nutrient agar plate preferentially entered into sporulation. Under these culture conditions, morphological changes during sporulation could be observed in detail with use of a video camera and were recorded by time lapse VTR. These observations were as follows.

About 16-24 hrs after it was placed on a non-nutrient agar plate, the sclerotium gradually changed into the plasmodium. After the change was completed, the plasmodium began to move on the agar plate and spread out with a network of plasmodial strands from its corner to the opposite side leaving a slime track. About $72 \mathrm{hrs}$ after the sclerotium was placed on the agar plate, plasmodium migration stopped, and protoplasm began to flow backwards, against the direction of plasmodium migration. After about 74 hrs, many projections were formed along the plasmodial strands; these projections increased in size and finally changed into discrete protoplasmic knobs (Figs. 1A-C). At this stage (the protoplasmic balling stage), the protoplasmic knobs were about $0.5-2 \mathrm{~mm}$ in diameter. After about $75.5 \mathrm{hrs}$, a papilla was projected at the apical region of each protoplasmic knob (Fig. 1D), gradually elongating upwards until it became a stalk (Fig. 1E). The stalk was formed with a rhythmical protoplasmic movement of about $1 \mathrm{~min}$ periodicity. Protoplasmic movement persisted synchronously at apical and basal regions of the stalk and non-synchronously between these two regions and the middle region. When the protoplasm contracted at the apical and basal regions of the stalk, it swelled at the middle region. During this time the stalk shrank in length and swelled in width. While the protoplasm swelled synchronously at the apical and basal regions of the stalk, it contracted at the middle region. After this the satlk grew in length and shrank in width. Stalk elongation lasted about $1 \mathrm{hr}$ (stalk elongation stage) (Fig. 1E). The apical part of the stalk began to expand 10 min before stalk elongation reached its maximum point (Fig. 1F) and changed into the sporangium head. The expansion of the head continued for $1 \mathrm{hr}$ (head expanding stage) (Fig. 1G). The middle and basal parts of the stalk became the stalk. The phase of protoplasmic movement between the head and stalk was reversed. Protoplasmic movement in the stalk stopped 30 min after the maximum elongation point was reached, and after a further $30 \mathrm{~min}$ it stopped in the head. The sporangium was formed with expansion of the head and shrinkage of the other parts of the stalk (Fig. 1G). The morphology of the sporangium underwent no remarkable change for $3 \mathrm{hrs}$ after the protoplasmic streaming had stopped (resting stage) (Fig. 1H). After the resting stage, the yellow sporangium started blackening rapidly, and after $30 \mathrm{~min}$ the head of sporangium was totally black (head blackening 
stage) (Fig. 1I) and the sporangium was morphologically completed (mature stage). Spores were formed gradually from mid-resting to mature stage, after cytoplasmic cleavage occurred at mid-resting stage. These events at various stages during the development of a sporangium
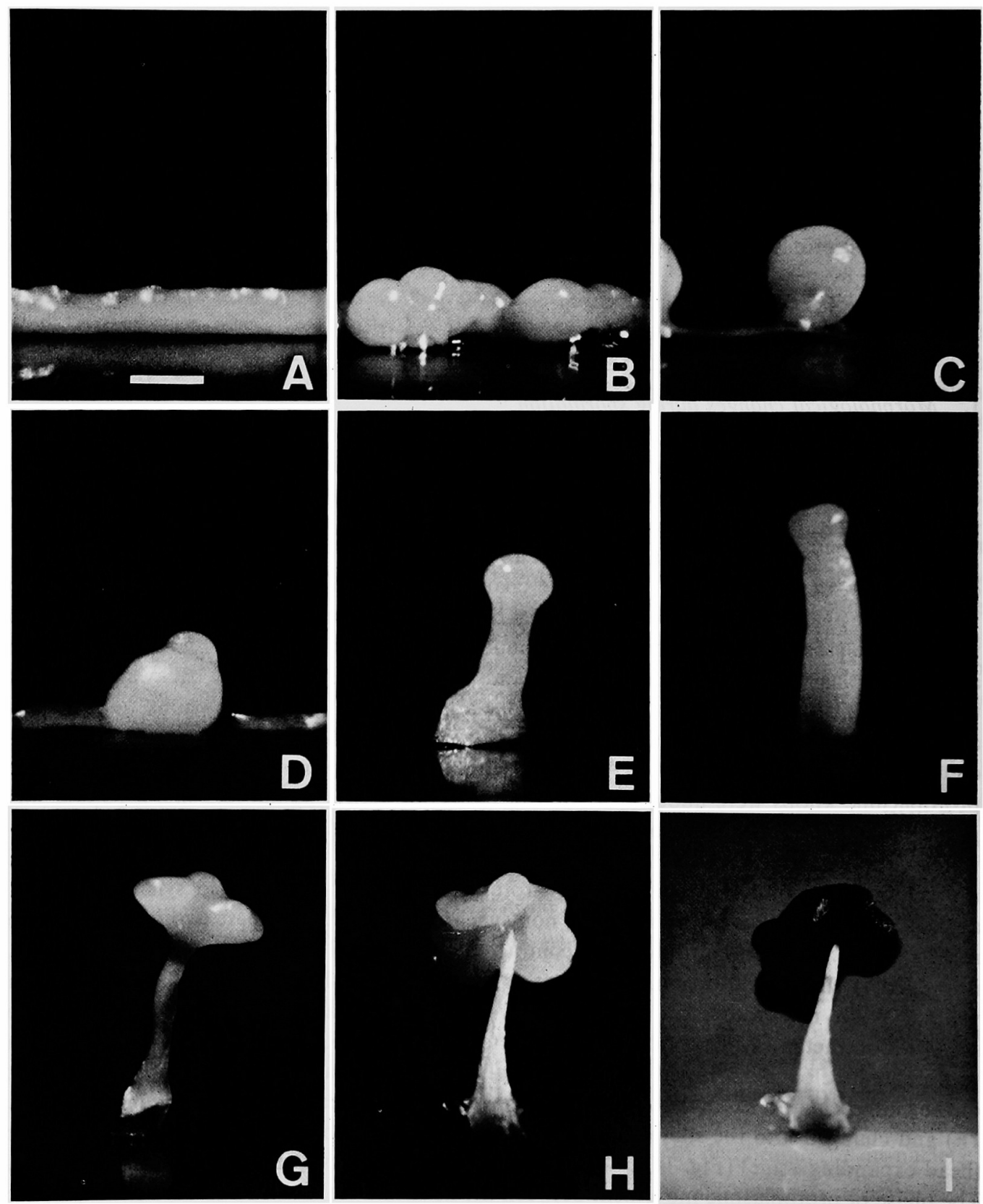

Fig. 1. Light micrographs of representative stages during sporulation in Physarum polycephalum. A, starved plasmodium reactivated from sclerotium. B, many projections formed along plasmodial strand after initiation of sporulation. C, protoplasmic knobs. D, a papilla projected from the apical region of a protoplasmic knob. E, a papilla gradually elongating upward. F, stalk elongation maximum point. G, a sporangium forming with expansion of apical part and shrinking of other parts in the stalk. $\mathrm{H}$, a sporangium at the resting stage. I, blackening of sporangial head. 
are summarized in the upper part of Fig. 8.

2. Behavior of cell nuclei

Cell nuclei in the sclerotium contained many heteropycnotic bodies (Fig. 2A), but during the starved plasmodium stage the chromatin in cell nuclei was diffuse (Fig. 2B). Cell nuclei in
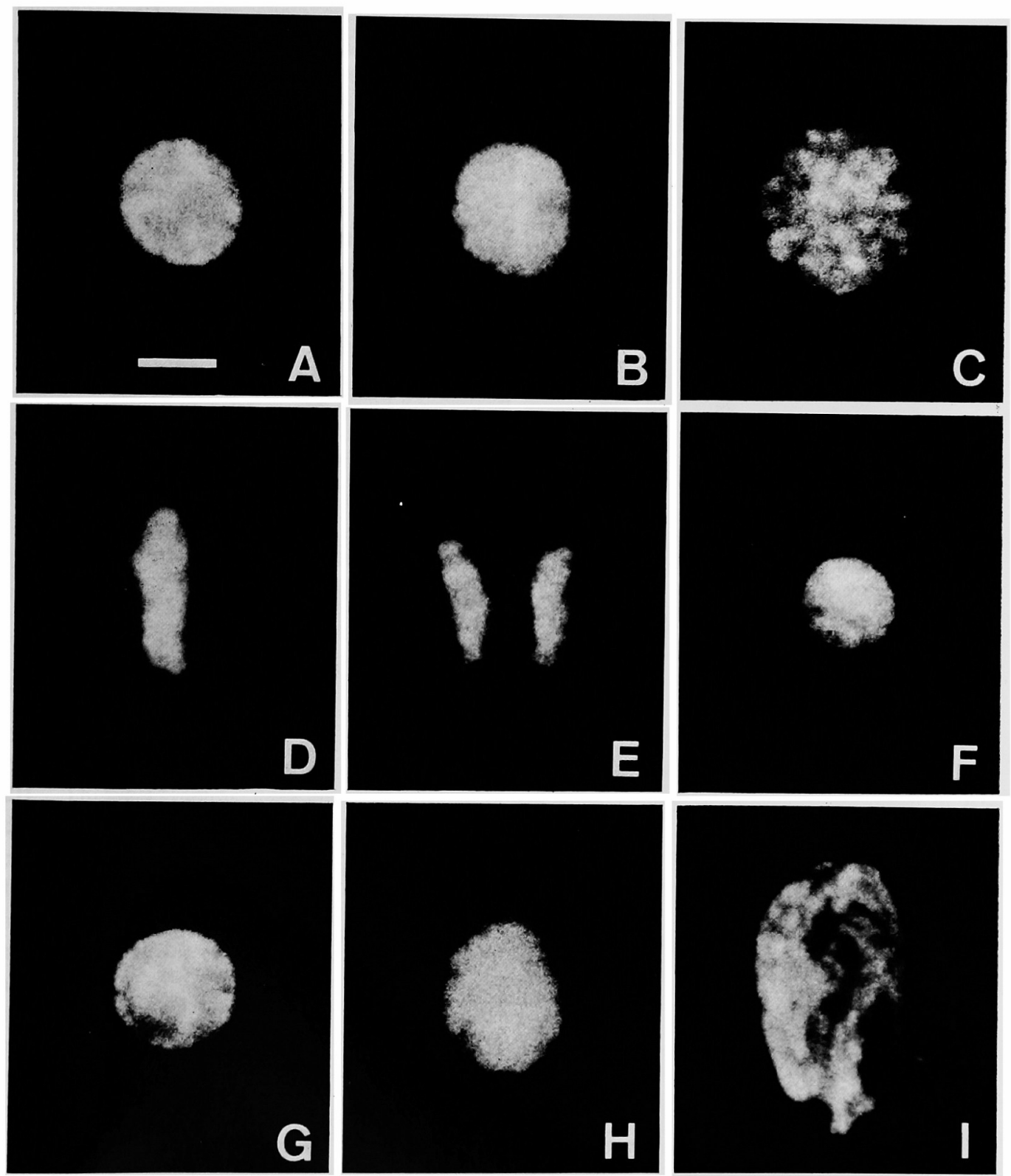

Fig. 2. Epifluorescence micrographs of cell nuclei at sclerotium, starved plasmodium and different stages of sporulation in $\boldsymbol{P}$. polycephalum. A cell nucleus at sclerotium (A) and starved plasmodium (B). Only a single mitosis was observed at resting stage of sporulation (C-E). Prophase (C) at early resting stage of sporulation, metaphase (D) and anaphase (E) at mid-resting stage of sporulation. A cell nucleus at late resting stage $(F$ and $G)$, mature stage $(H)$ and meiotic prophase $I(I)$ at mature stage of sporulation. $B a r=2 \mu \mathrm{m}$. 

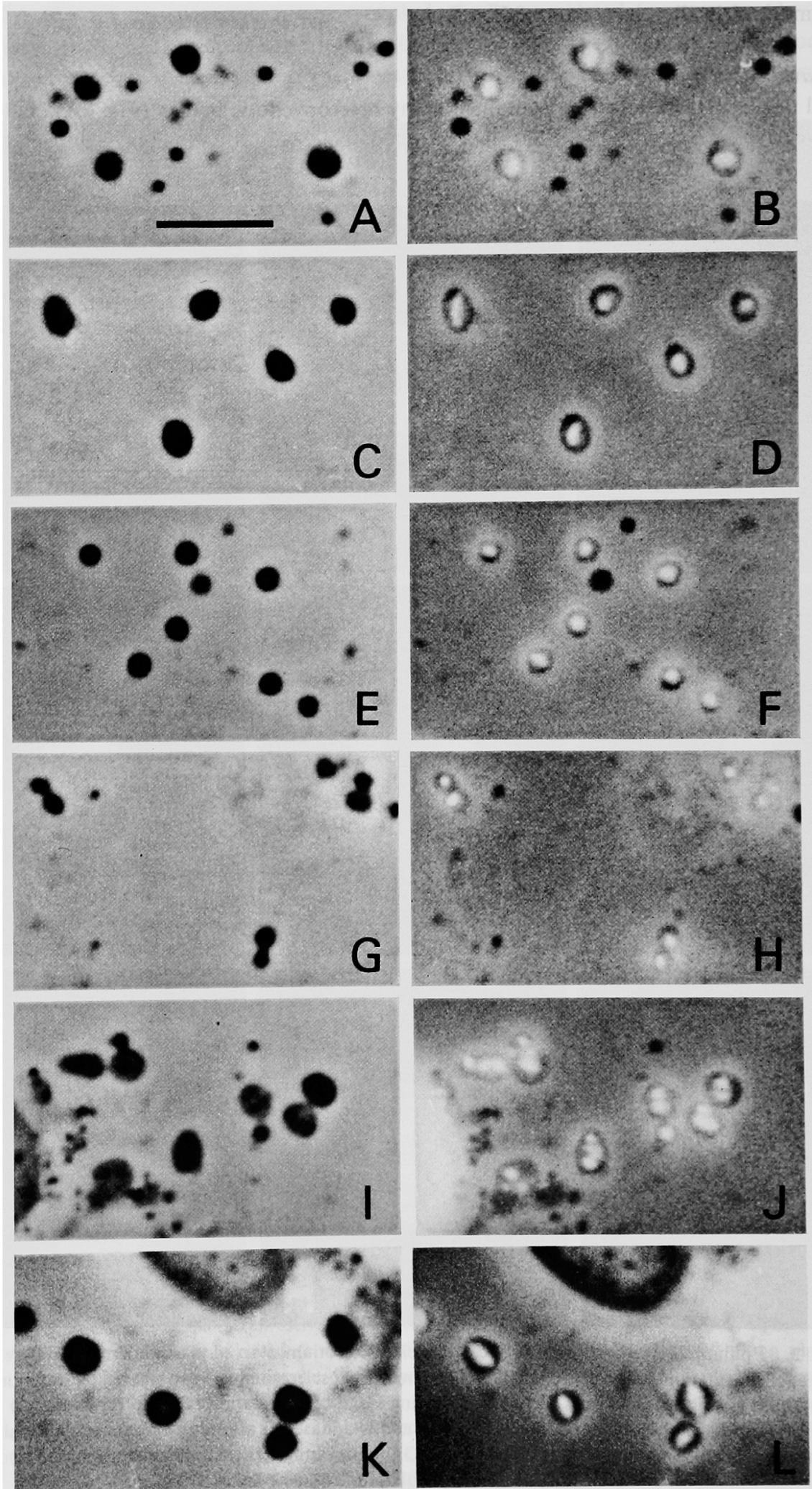
sclerotium were similar in size to those of plasmodium $(3.0-3.7 \mu \mathrm{m}$ in minor axis and 3.4 $4.2 \mu \mathrm{m}$ in major axis). Cell nuclear division could not be seen by observation at intervals of $1 \mathrm{hr}$ during the starved plasmodium stage. After sporulation started only a single somatic cell nuclear division occurred synchronously, at the resting stage of sporulation (Figs. 2C-E). Cell nuclei entered into mitotic prophase (Fig. 2C) at the early resting stage, persisted in prophase for about $1.5 \mathrm{hrs}$, and passed through metaphase (Fig. 2D), anaphase (Fig. 2F) and telophase for a very short time $(15-30 \mathrm{~min})$. After nuclear division nuclei became small (2.6-3.0 $\mu \mathrm{m}$ in diameter) (Fig. $2 \mathrm{~F}$ ) but increased in size from the late resting (Fig. 2G) and early mature stages (Fig. 2H). During these stages many heteropycnotic bodies appeared in the nuclei. Cytoplasmic cleavage occurred around each nucleus after nuclear division at the midresting stage, and spores were formed during the mature stage. The chromatin in cell nuclei

Table 1. Relative DNA content of cell and mitochondrial nuclei at sclerotia and different stages of starved plasmodia and sporulation in $P$. polycephalum, using DAPI epifluorescence microsperctrophotometry, measured in 100 cell nuclei and mt-nuclei at each stage

\begin{tabular}{cccc}
\hline & & \multicolumn{1}{c}{$\begin{array}{c}\text { Fluorescence intensity } \\
\text { (arbitrary unit) }\end{array}$} \\
\cline { 2 - 3 } Stage & Time (hr) & Cell nucleus & Mt-nucleus \\
\cline { 2 - 4 } Sclerotium & 0 & $79.9 \pm 6.3$ & $18.2 \pm 5.7$ \\
Plasmodium early & 20 & $77.5 \pm 7.2$ & $17.3 \pm 5.1$ \\
mid & 40 & $77.3 \pm 4.6$ & $8.5 \pm 2.5$ \\
late & 60 & $79.0 \pm 5.8$ & $9.1 \pm 2.9$ \\
Sporangium formation & & & $8.7 \pm 2.6$ \\
Resting early & 79.5 & $75.4 \pm 5.5$ & $8.6 \pm 2.5$ \\
mid & 80.5 & $40.3 \pm 4.7$ & $9.0 \pm 2.6$ \\
late & 81.5 & $57.8 \pm 12.2$ & $8.3 \pm 2.3$ \\
Mature & 84.5 & $78.1 \pm 6.6$ & $8.2 \pm 2.4$ \\
Meiotic prophase I & 106 & $76.7 \pm 4.8$ & $79.3 \pm 5.2$ \\
Mitotic G2 (control) & & & \\
\hline
\end{tabular}

became diffuse during the mature stage (Fig. $2 \mathrm{H}$ ). After about $24 \mathrm{hrs}$ from initiation of head blackening, cell nuclei entered into early prophase and rested at this stage within spores (Fig. 2I). This stage seems to be meiotic early prophase I, according to the previous report of Al- * drich (1967). DAPI epifluorescence microspectrophotometry showed that cell nuclear DNA content was constant from the sclerotium stage to the early resting stage of sporulation (Table 1). At these stages, cell nuclear DNA content was similar to that in somatic diploid G2 phase nuclei (Table 1). After initiation of sporulation, when cell nuclei divided into daughter ones, nuclear DNA content decreased by one half. After nuclear division, nuclear DNA immediately synthesized twofold within 4 hrs. After doubling of nuclear DNA content, it remained constant up to meiotic prophase I (Table 1). These events of cell nuclei are summarized in the middle part of Fig. 8.

Fig. 3. Phase contrast (A, C, E, G, I and K) and phase contrast fluorescence (B, D, F, H, J and L) photographs of mitochondria and mt-nuclei at sclerotium, starved plasmodium and different stages of sporulation and at amoeba stage in $P$. polycephalum after DAPI staining. Spherical or oval mitochondria at sclerotium (A) and starved plasmodium (C). A spherical or oval mt-nucleus can be seen as a brilliant spot in each mitochondrion at sclerotium (B) and starved plasmodium (D). $\mathrm{E}$, diminutive mitochondria at mid-stage of starved plasmodium. $\mathrm{F}$, diminutive mitochondria contain one small spherical or oval mt-nucleus. G, dumbbell-shaped mitochondria at resting stage of sporulation. $\mathrm{H}$, note that dumbbell-shaped mitochondria contain two nuclei, one in each side of the dumbbell. I and $\mathrm{J}$, multinucleated mitochondria at meiotic prophase $\mathrm{I}$. $\mathrm{K}$ and $\mathrm{L}$, mitochondria in an amoeba contain only one mt-nucleus. Bar $=5 \mu \mathrm{m}$. 


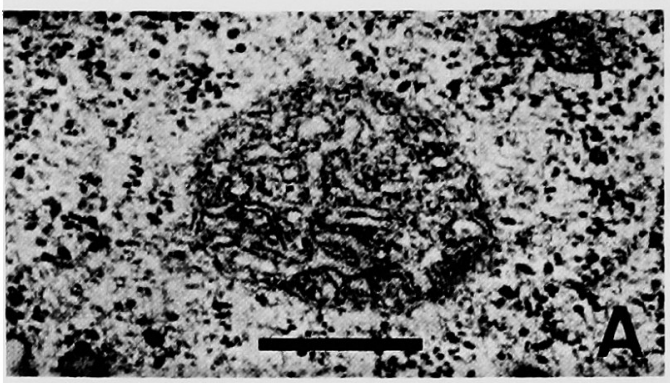

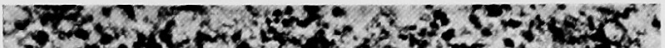

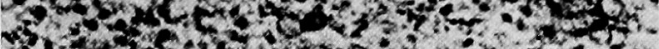

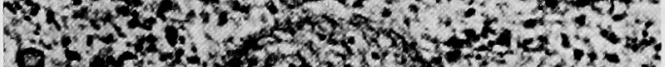

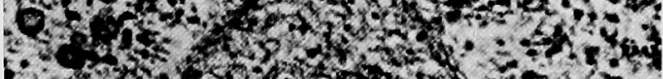

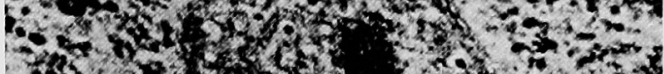

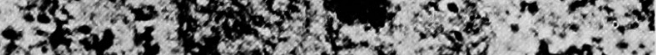

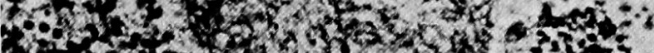

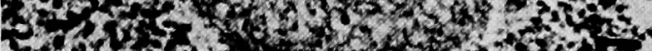

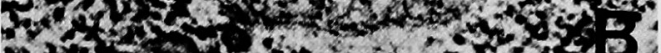

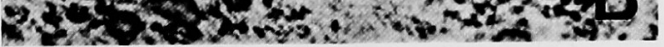

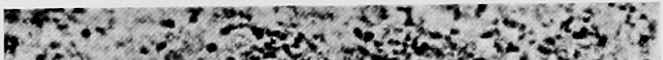

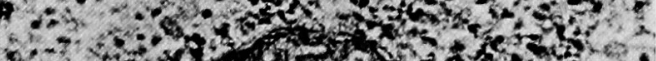

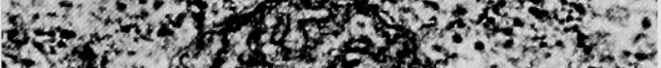

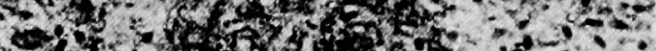

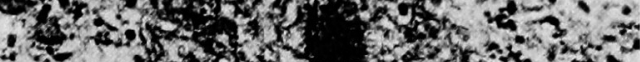

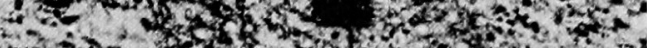

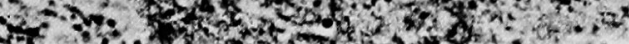

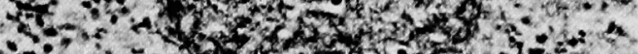

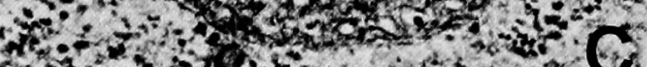

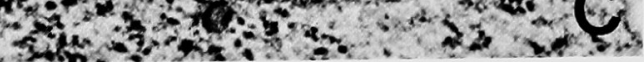

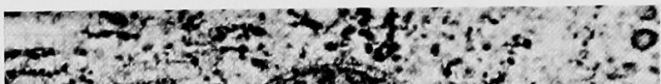

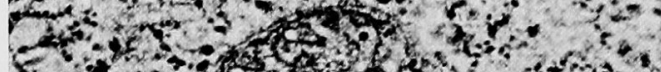

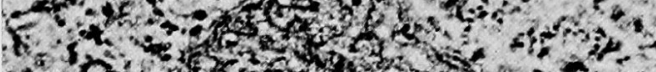

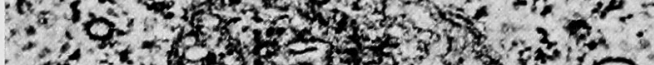

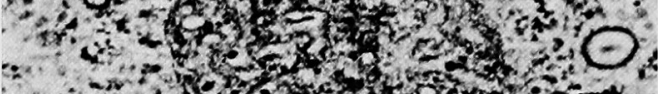

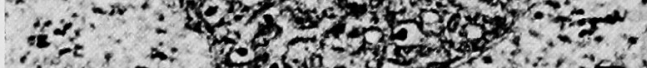

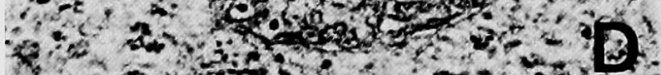

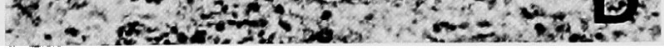

Fig. 4. Electron micrographs of part of the ultrathin serial sections of a mitochondrion at the protoplasmic balling stage of sporulation in $P$. polycephalum. Figs. A, B, C and D correspond to the fourth, fifth, sixth and seventh sections of ten serial sections. A single electron-dense mt-nucleus can be seen in the center of the mitochondrial body. $\operatorname{Bar}=1 \mu \mathrm{m}$.
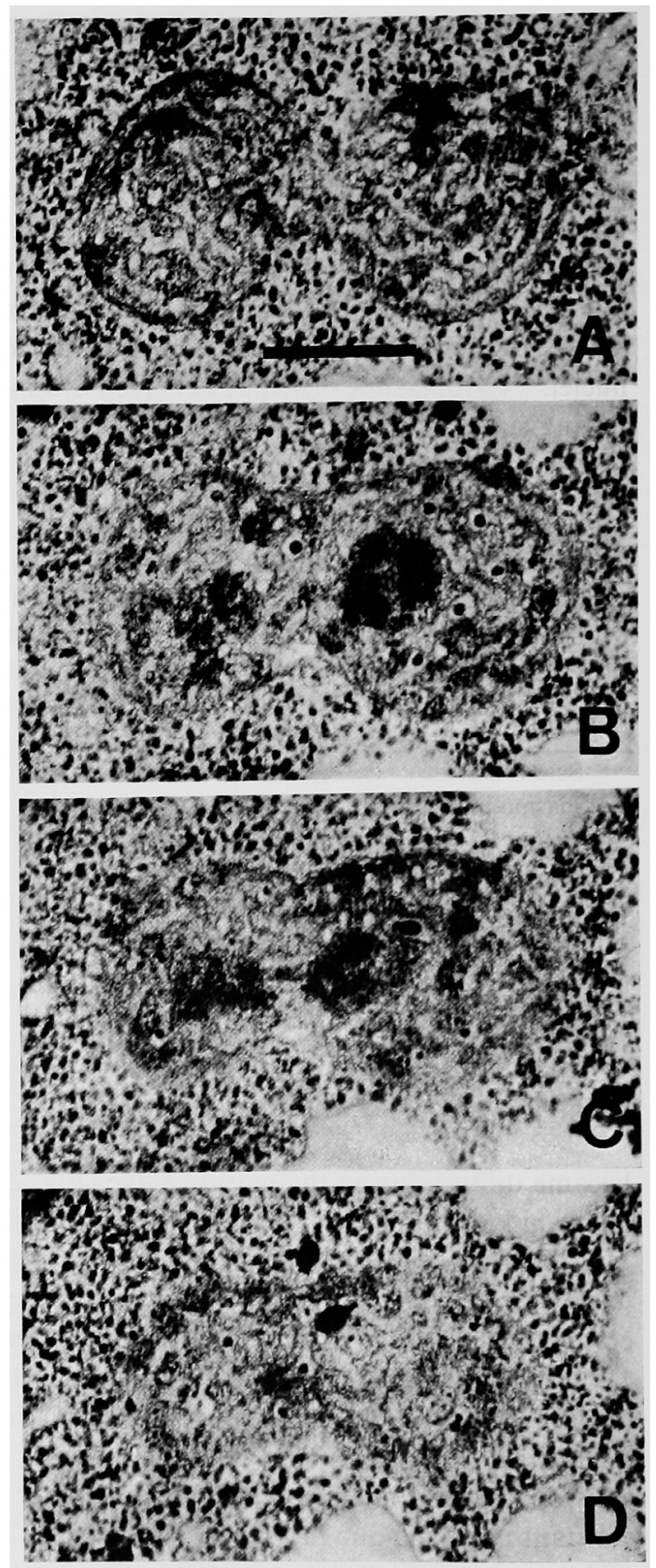

Fig. 5. Electron micrographs of part of the ultrathin serial sections of a dumbbell-shaped mitochondrion at mid-resting stage of sporulation in $P$. polycephalum. A, B, C and D correspond to the fourth, sixth, seventh and eighth sections of 9 serial sections. The dumbbell-shaped mitochondrion cntains two nuclear areas composed of some electron-dense and semi-electron-dense materials. Each of two nuclear areas is located in each side of the dumbbell. $\mathrm{Bar}=1 \mu \mathrm{m}$. 


\section{Behavior of mitochondria}

The protoplasm of sclerotium was surrounded by a cell wall. About 20 mitochondria per cell nucleus were observed in the sclerotium. Mitochondria were spherical or oval during sclerotium and early resting stages (Figs. 3A-D). Mitochondria were $1.0-1.5 \mu \mathrm{m}$ in minor axis and $1.2-1.8 \mu \mathrm{m}$ in major axis at sclerotium and early starved plasmodium stages but became small at mid-starved plasmodium stage $(0.9-1.1 \mu \mathrm{m}$ in minor axis, $0.9-1.2 \mu \mathrm{m}$ in major axis) (Figs. 3E and F). Mitochndria remained small from mid-starved to early resting stage. When cytoplasmic cleavage occurred at mid-resting stage, mitochondria acquired dumbbell or irregular shapes (Fig. 3G). The dumbbell- and irregular-shaped mitochondria became spherical or oval at the late resting and early mature stages. Mitochondria rested with spherical or oval shape at meiotic prophase I in a spore (Fig. 3I). About 10 mitochondria per spore were observed in mature spores.

Table 2. Number of nuclei per mitochondrion at sclerotia and different stages of starved plasmodia and sporulation in P. polycephalum after DAPI staining, counted in 500 mitochondria at each stage

\begin{tabular}{lccccccc}
\hline \multirow{2}{*}{ Stage } & Time (hr) & \multicolumn{6}{c}{ Number of nuclei per mitochondrion } \\
\cline { 3 - 7 } & & 1 & 2 & 3 & 4 & 5 & 6 \\
\hline Sclerotium & 0 & 92.0 & 7.2 & 0.6 & 0.2 & & \\
Plasmodium early & 20 & 98.9 & 1.1 & & & & \\
mid & 40 & 98.8 & 1.2 & & & & \\
late & 60 & 98.1 & 1.9 & & & & \\
Sporangium formation & & & & & & & \\
Resting early & 79.5 & 94.9 & 4.8 & 0.2 & & & \\
mid & 80.5 & 83.6 & 14.6 & 1.9 & & & \\
late & 81.5 & 57.6 & 32.0 & 7.4 & 1.7 & 1.2 & 0.2 \\
Meiotic prophase I & 106 & 55.1 & 35.4 & 7.7 & 1.8 & & 0.2 \\
\hline
\end{tabular}

\section{Behavior of $m t$-nuclei}

DAPI-stained mt-nuclei were observed as brilliant-blue spots within mitochondrial bodies, as shown in phase contrast fluorescence photographs (Figs. 3B, D, F, H, J and L). At the sclerotium stage many mitochondria contained one mt-nucleus, but multinucleated mitochondria were observed at low frequency (Table 2). At the reactivated plasmodium stage, multinucleated mitochondria decreased in frequency and most mitochondria contained a short rod or ovoid shaped mt-nucleus (Table 2). After entering into sporulation, dumbbell-shaped mitochondria appeared at the resting stage (Fig. 3G). Dumbbell-shaped mitochondria contained two nuclei (Fig. $3 \mathrm{H}$ ), and one mt-nucleus was located in each side of the dumbbell. During the resting stage, multinucleated mitochondria increased in frequency. At the late resting stage of sporulation mitochondria which contained four, five and six nuclei appeared at low frequency (Table 2). The number of mt-nuclei per mitochondrion and their frequency remained unchanged from the late resting stage to meiotic prophase I (Table 2). Mt-nuclei were spherical or oval from sclerotium to mature stage of sporulation (Figs. 3B, D, F, H and J). The size of the mt-nuclei was $0.3-0.6 \mu \mathrm{m}$ in minor axis and $0.5-1.2 \mu \mathrm{m}$ in major axis at sclerotium and early starved plasmodium stage (Figs. 3B and D). Mt-nuclei were smaller at the mid-starved plasmodium stage than at the sclerotium and early starved plasmodium stages (Figs. 3A-F). The size of mitochondrial nuclei at mid-starved plasmodium stage was $0.3-0.5 \mu \mathrm{m}$ in minor axis and 0.4 $0.7 \mu \mathrm{m}$ in major axis. It was constant during the mid-starved plasmodium stage and meiotic prophase I. As shown in Table 1, mt-nuclear DNA content per mitochondrion was constant during sclerotium and early starved plasmodium stage, but decreased by one half at the mid- 

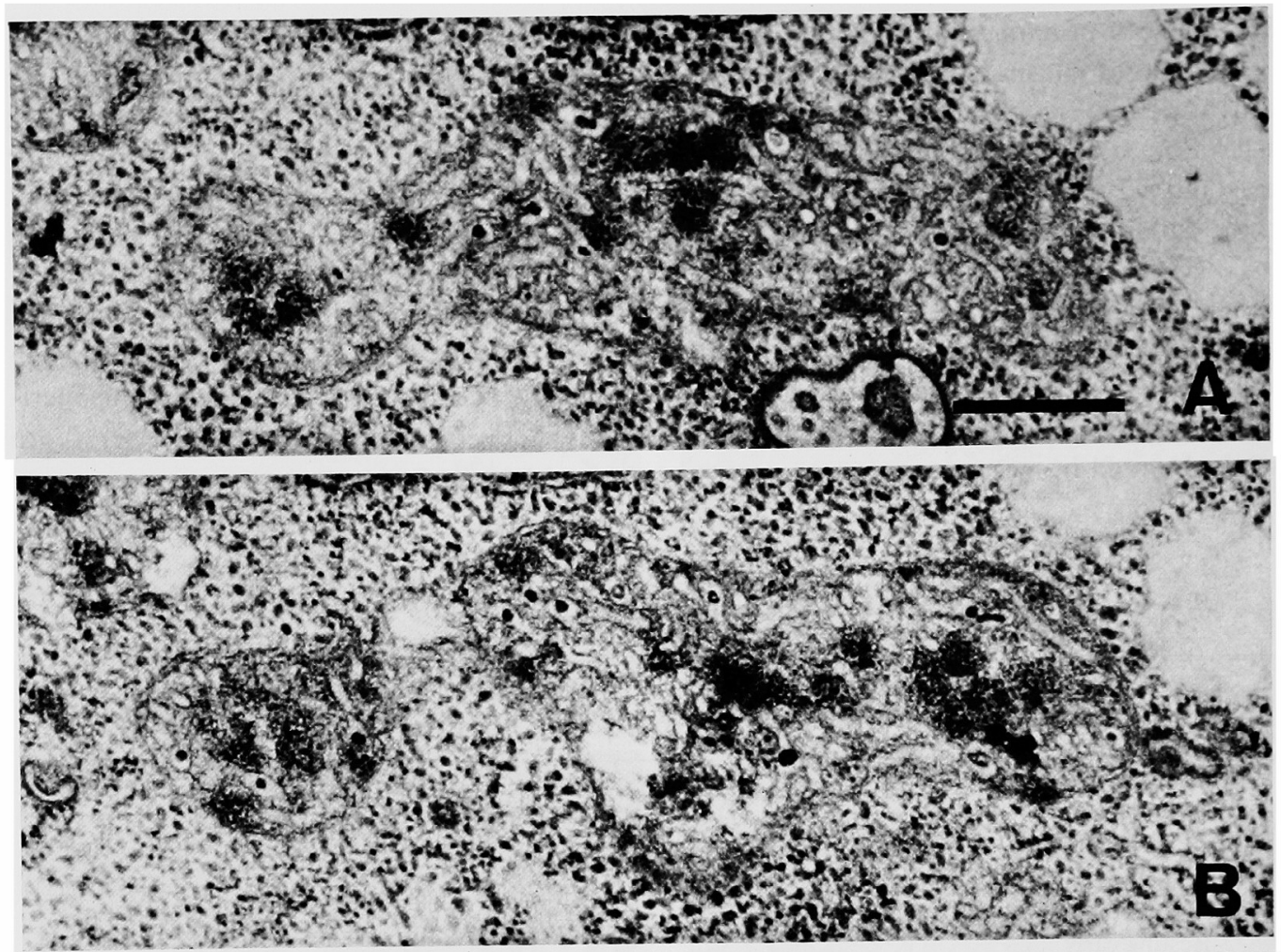

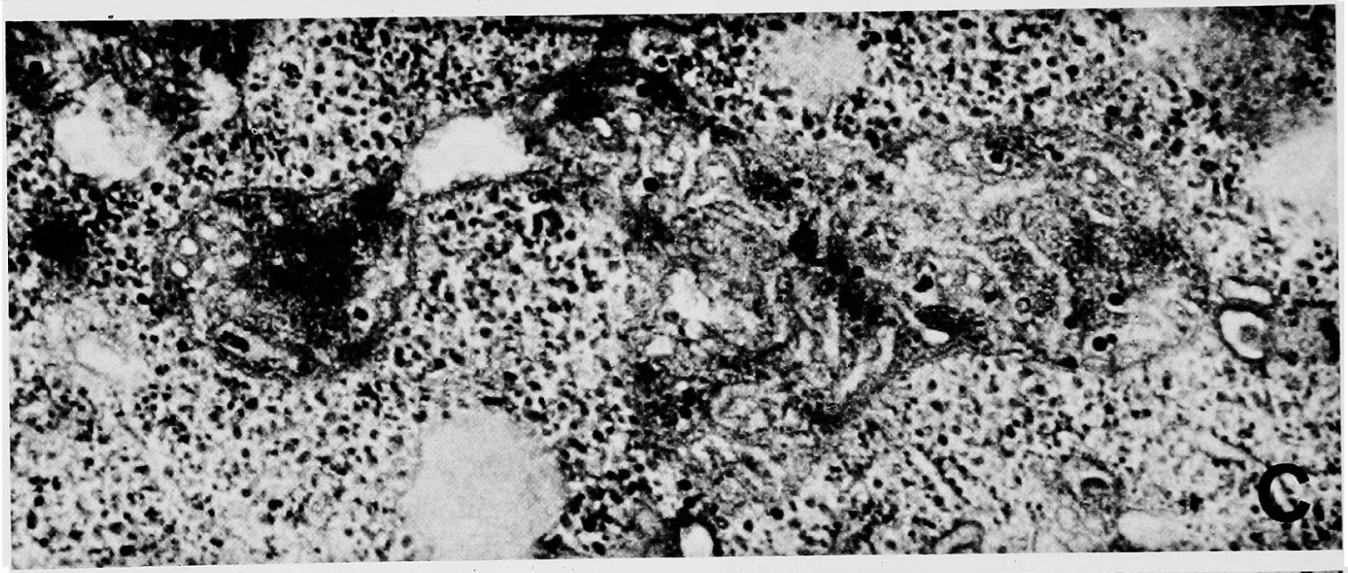

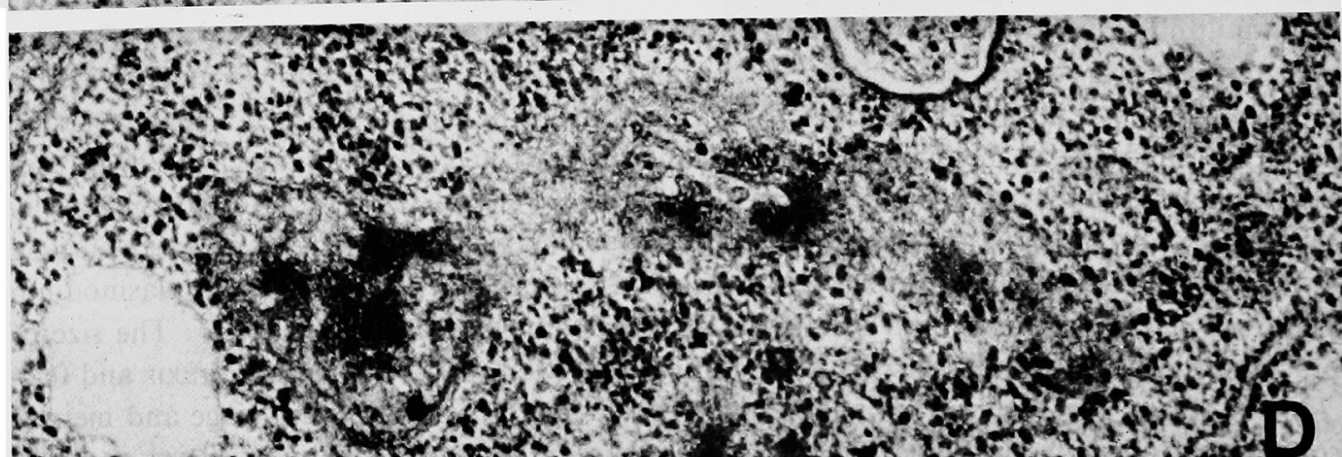

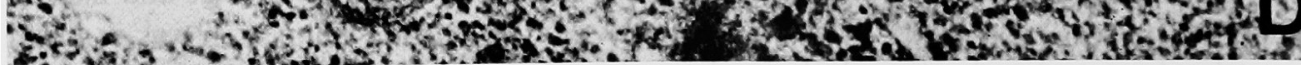


starved plasmodium stage and remained the same from mid-starved plasmodium stage to premeiotic division stage. The total fluorescence intensity of a few mt-nuclei in multinucleated mitochondria increased stepwise with the number of mt-nuclei. These results suggest that a few mt-nuclei in multinucleated mitochondria partially attach or fuse at the mature stage. On the other hand, the mitochondria in amoebae which originated from spores contained only one mt-nucleus (Figs. $3 \mathrm{~K}$ and $\mathrm{L}$ ). It may be suggested that mitochondrial nuclear fusion occurs at some stage between spore germination and the amoeba stage.
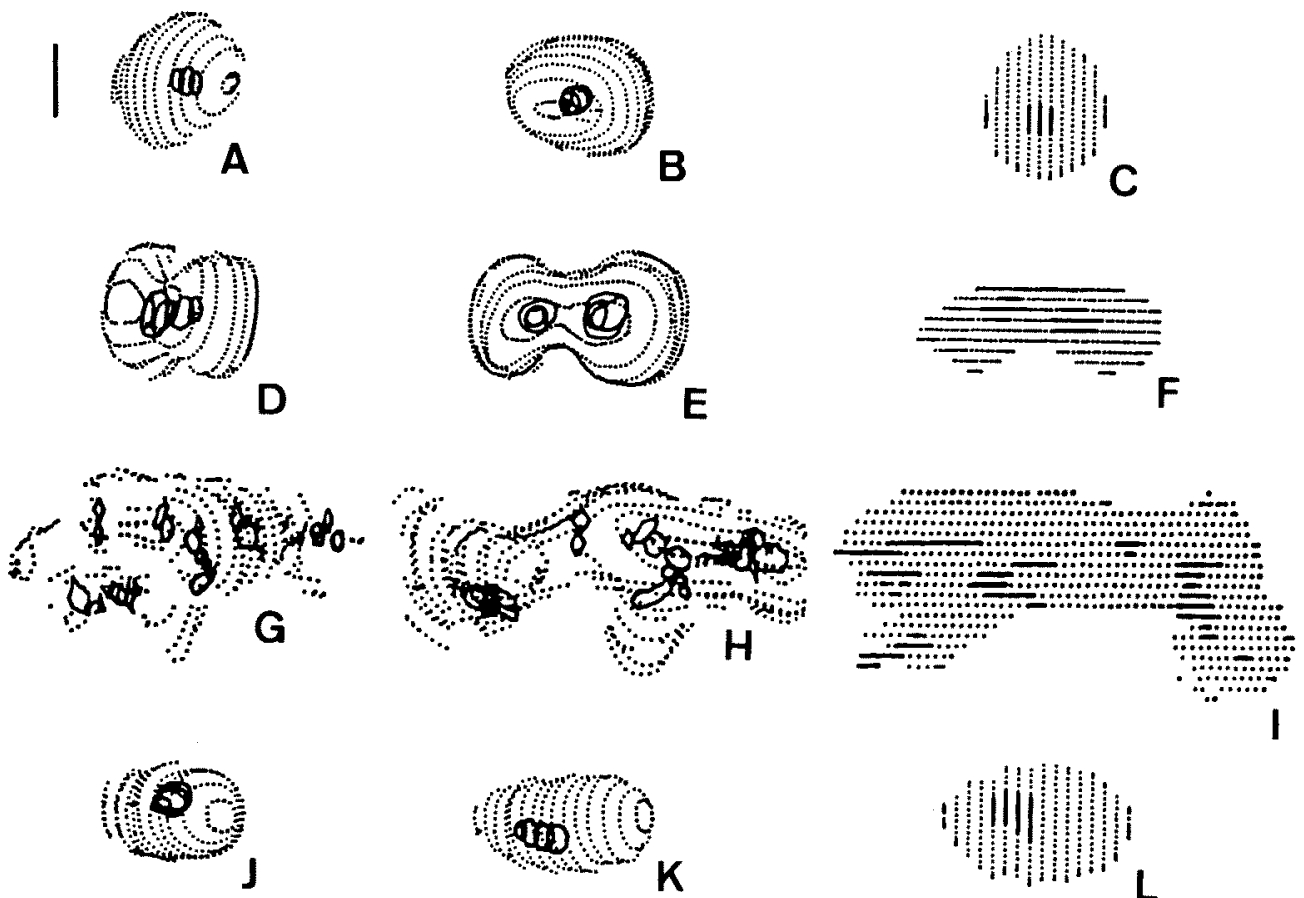

Fig. 7. Three-dimensional profile of mitochondria and mt-nuclei at protoplasmic balling stage (A-C), mid-resting stage (D-I), amoeba stage ( $\mathrm{J}-\mathrm{L}$ ) corresponding to Figs. 4, 5, 6 and 7, respectively, reconstructed by computer-aided graphics. For each stage three side views of one mitochondrion with $\mathrm{mt}$-nuclei are shown. Dotted and full lines show outline of mitochondrial bodies and $\mathrm{mt}-$ nuclear areas, respectively. $\mathrm{Bar}=1 \mu \mathrm{m}$.

\section{Electron microscopic observations of mitochondria and their nuclei}

The observations of mitochondria and mitochondrial nuclei by ultra-thin serial sections are shown in Figs. 4-7.

A spherical electron-dense mt-nucleus about $0.5 \mu \mathrm{m}$ in diameter was observed in the central portion of the mitochondrion during the protoplasmic balling stage (Figs. 4B-C and 7A-C). Electron-dense calcium deposition was contained in the tubulous cristae of mitochondria during sporulation (Figs. 4-6), as previously reported (Daniel and Jarlfors 1972, Nicholls 1972). At mid-resting stage dumbbell- (Figs. 5A-D) or irregular-shaped (Figs. 6A-D) mitochondria were observed. At this stage, mt-nuclear structure changed; mt-nuclei were composed irregularly

Fig. 6. Electron micrographs of part of the ultra-thin serial sections of an irregular-shaped mitochondrion at the mid-resting stage of sporulation in P. polycephalum. A. B, C and D correspond to the sixth, ninth, tenth and twentieth section of 21 serial sections. The irregularly shaped mitochondrion contains at least four or five nuclear areas composed of some electron-dense and semi-

electron-dense materials. Bar $=1 \mu \mathrm{m}$. 


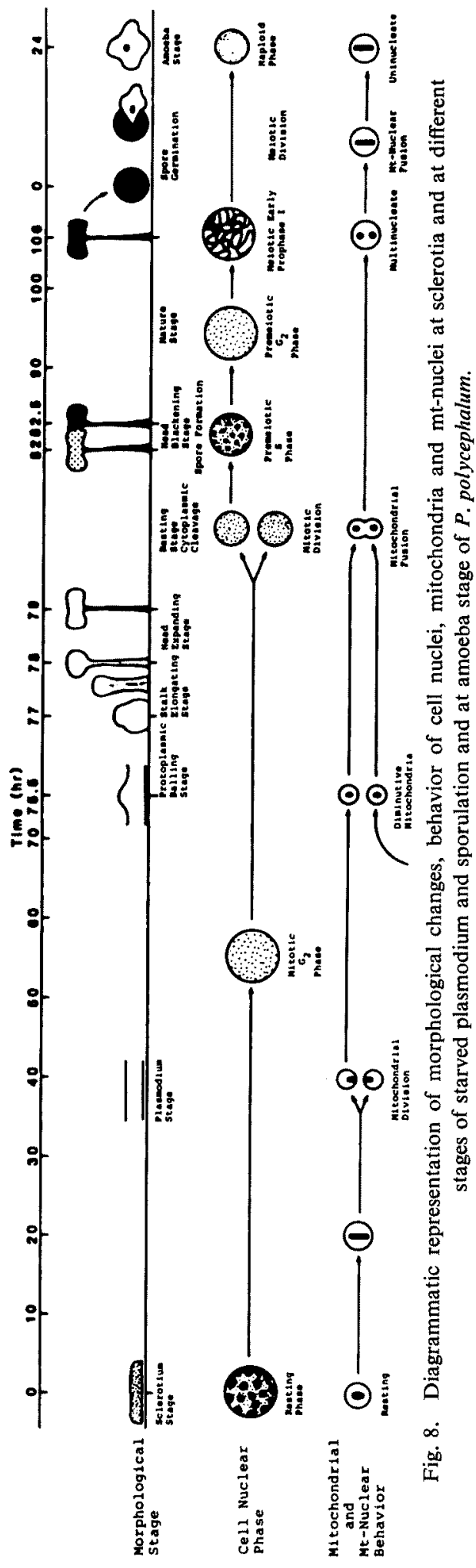

of some electron-dense and semi-electrondense materials, and they beacame amorphous (Figs. 5B-C and 6A-D). However, three-dimensional reconstruction profiles of mitochondria and mt-nuclei by computeraided graphics revealed a dumbbell-shaped mitochondrion contained at least two nuclear areas consisting of electron-dense and semielectron-dense materials (Figs. 7D-F), and that an irregularly shaped mitochondrion contained at least four or five nuclear areas (Figs. 7G-I). In the dumbbell-shaped mitochondrion one mt-nuclear area was located in each side of the dumbbell (Figs. 7D-F). On the other hand, three-dimensional reconstruction profile by computer-aided graphics showed that in amoeba the mitochondrion contained only one electron-dense nucleus (Figs. 7J-L). These electron microscopic observations show that multinucleated mitochondria appear at the resting stage of sporulation, as observed in light microscopy (Figs. $3 \mathrm{H}$ and $\mathrm{J}$ ), and suggest that mitochondrial nuclear fusion occurs at some stage between spore germination and the amoeba stage, following mitochondrial fusion at the resting stage of sporulation (Fig. 8).

\section{Discussion}

\section{Morphological changes during sporulation}

Morphological changes during sporulation in Physarum polycephalum have been previously observed by Guttes et al. (1961). The present study by night vision camera and time lapse VTR further clearly showed continuous morphological changes and time lapse during sporulation in $P$. polycephalum (Figs. 1 and 8). Morphogenesis of sporulation in $P$. polycephalum consists largely of three dramatic events: protoplasmic balling, stalk formation and elongation, and head formation of sporangium. These morphogenetic movements are sequential and are accompanied by periodic protoplasmic movements before the resting stage, as in proliferating macroplasmodia (Kamiya 1940, Kamiya and Seifriz 1954). 


\section{Behavior of cell nuclei}

Nuclear DNA content in sclerotia of $P$. polycephalum is the same as in diploid G1 phase nuclei, as determined by Burton's diphenylamine reaction method (Mohberg and Rusch 1971). In the present study nuclear DNA content in sclerotia was the same as in somatic diploid G2 phase nuclei (Table 1). In our culture conditions, spherulation is induced rapidly for 1-2 days from macroplasmodia, while in the culture conditions used by Mohberg and Rusch (1971) it is done slowly for about $\mathbf{2 0}$ days from macroplasmodia. It is well-known that culture conditions affect nuclear behavior, as observed by endopolyploidy, aneuploidy, extra DNA amplification, etc. in cultured cells of higher plants (D'Amato 1977, Nagl 1978). The sclerotia we used may be similar to the starved plasmodia used by Mohberg and Rusch (1971), since they have similar cell nuclear DNA content. Macroplasmodium from a suspension of microplasmodia is readily by starvation for 4 days before illumination to induce sporulation (Sauer et al. 1969). Starved macroplasmodia have a DNA content close to that of diploid G2 phase nuclei, as determined with the use of the Burton method (Mohberg and Rusch 1971) and by Feulgen spectrophotometry (Arescaldino 1971). Results of ${ }^{3} \mathrm{H}$-thymidine incorporation into DNA soluble fractions in hot perchloric acid have indicated that DNA synthesis occurs before illumination to induce sporulation. This means that at least one mitosis occurs before DNA synthesis (Sauer et al. 1969).

Starved macroplasmodia from sclerotia contains the same amount of DNA as somatic diploid G2 phase nuclei (Table 1). Mitosis was not observed in the starved plasmodia in the interval of $1 \mathrm{hr}$ (Fig. 2). The starved plasmodia from sclerotia may be physiologically different from those reported previously (Sauer et al. 1969), because of the different culture conditions. After entering into sporulation only a single somatic nuclear division was observed, at the resting of sporulation, as previously shown by electron microscopy (Aldrich 1967). After nuclear division cytoplasmic cleavage occurred around each nucleus and nuclear DNA immediately synthesized twofold within $4 \mathrm{hrs}$ (Table 1). This nuclear DNA synthesis seems to be premeiotic, on the basis of previous electron microscopic observations (Aldrich 1967). Cell nuclei passed through long premeiotic G2 phase (about $24 \mathrm{hrs}$ ), entered into meiotic prophase I and rested in this stage in mature spores (Figs. 2 and 8 ). At the time that spores germinate meiotic division occurs, and amoebae which originate from spore germination are haploid by chromosome count and estimation of nuclear DNA content (Mohberg et al. 1973).

\section{Behavior of mitochondria and mt-nuclei}

In $P$. polycephalum, during spherulation from starved suspended microplasmodia in liquid medium, mitochondria decreased in size and diminutive mitochondria contained very small mt-nuclei (Kawano et al. 1983). The DNA content per mitochondrion decreased in accordance with the decrease in mitochondrial and mt-nuclear size. However, endonuclease Eco R1 and Bst N1 fragment patterns showed that the physical structure of mt-DNA molecules in $P$. polycephalum did not change during spherulation as well as in microplasmodia. It is suggested that in $P$. polycephalum, a reduction in the number of whole mitochondrial genomes occurs by mitochondrial division not accompanying DNA synthesis during spherulation (Kawano et al. 1983).

Also in the present study, mitochondria decreased in size between early and mid-stages of starved plasmodia (Figs. 3E and F). The DNA conetnt per mitochondrion decreased by one half on the average between early and mid-stages of starved plasmodia (Table 1). The production of diminutive mitochondria in starved plasmodia is similar to that during spherulation. It may be considered that diminutive mitochondria result in mitochondrial division not accompanying DNA synthesis, as suggested in mitochondria during spherulation (Kawano et al. 1983). Dumbbell-shaped mitochondria appeared at the resting stage of sporulation, and con- 
tained two discrete mt-nuclei (Figs. 3G and $\mathrm{H}$ ). The two nuclei were located in each side of the dumbbell-shaped mitochondrion. The dumbbell-shaped mitochondria at this stage differ from dumbbell-shaped mitochondria containing a dumbbell-shaped mt-nucleus in proliferating microplasmodia (Kuroiwa et al. 1977). During the resting stage of sporulation dumbbellshaped or multinucleated mitochondria increased in frequency (Table 2). DNA content per mt-nucleus in the dumbbell-shaped or multinucleated mitochondria was similar to that in mitochondria containing a single nucleus before the resting stage (Table 1). On the other hand, the number of mitochondria per cell nucleus decreased during the resting stage. These data suggest that at the resting stage of sporulation the formation of dumbbell-shaped or multinucleated mitochondria is due to mitochondrial fusion. Mitochondrial fusion has been observed in Saccharomyces cerevisiae in which many small spherical mitochondria fused into a single large and long mitochondrion at the latter stage of premeiotic S phase (Hoffman and Avers 1973, Sando et al. 1981, Stevens 1981, Miyakawa et al. 1984). Ultra-thin serial sections also showed the presence of dumbbell- or irregular-shaped mitochnodria which contained two and four or five mt-nuclei at the resting stage of sporulation (Figs. 5-7). The nuclear structure of mt-nuclei at the resting stage was dramatically changed from that before the resting stage. At the electron microscopic level, mt-nuclei were composed of compactly electrondense material before the resting stage (Fig. 4), but at the resting stage (Figs. 5 and 6) they consisted of irregularly electron-dense and semi-electron-dense materials. At the light microscopic level, however, the shape of mt-nuclei was clearly spherical or oval throughout the sporulation processes (Figs. $3 \mathrm{H}$ and $\mathrm{J}$ ). In actively proliferating plasmodia of $P$. polycephalum, mt-nuclei are composed of a semi-electron-dense axial material which is sensitive to DNase treatment, and an electron-dense peripheral material which surrounds the axial material and is sensitive to RNase treatment (Kuroiwa 1973).

At the resting stage of sporulation in $P$. polycephalum, the electron-dense material of the mt-nuclear area may be composed of RNA clusters, and it seems likely that electron-dense materials around the mt-nuclei are fragmented into some parts at the resting stage.

Using DAPI epiffuorescence microscopy it was observed that mt-nuclei were succeedingly fused after mitochondrial fusion and formed long threads with formation of thread-like giant mitochondria during the later stage of premeiotic $\mathrm{S}$ phase in meiotic cells of Saccharomyces cerevisiae (Sando et al. 1981, Miyakawa et al. 1984).

In the present studies, using epifluorescence microscopy and the serial sectioning technique, mt-nuclei in some mitochondria could be seen to partially attach or fuse at the mature stage after mitochondrial fusion (Fig. 3J). The present results confirmed that mt-nuclei also were fused following mitochondrial fusion.

\section{Summary}

The morphological behavior of cell nuclei, mitochondria and mitochondrial nuclei during sporulation in Physarum polycephalum was investigated using light and electron microscopy. The plasmodia which reactivated from sclerotia on non-nutrient agar plates preferentially entered into sporulation. A night vision camera and time lapse VTR were available to monitor morphological changes and determine the exact time course. Sporulation processes were classified into six stages, by morphological characteristics. The initial event of sporulation was characterized by the formation of many protoplasmic knobs along plasmodium strands (protoplasmic balling stage). A papilla was projected from the apical region of each protoplasmic knob, elongated and changed to form a stalk (stalk elongation stage). The apical part of the stalk then began to expand until it reached a maximum point of stalk elongation, thereafter differentiating into the head of the sporangium (head expanding stage). After a 
stage without morphological changes (resting stage), the color of the sporangial head gradually changed from pale yellow to black (head blackening stage). After full blackening of the sporangial head, sporangia were completed (mature stage). 4'6-diamidino-2-phenylindole (DAPI) epifluorescence microspectrophotometry showed that the cell nuclear DNA content in sclerotia and starved plasmodia before the resting stage was the same as that in somatic diploid G2 phase nuclei. Only a single somatic nuclear division occurred at mid-resting stage. Meiotic DNA synthesis then occurred during the late resting and head blackening stages, when cytoplasmic cleavages occurred. After DNA synthesis, cell nuclei persisted in a long G2 phase of about one day and stopped at meiotic prophase I. In sclerotia and starved plasmodia, mitochondria were spherical or oval and contained one electron-dense mitochondrial nucleus (mtnucleus). Mitochondria and mt-nucleus became smaller in size in starved plasmodia. The DNA content per mitochondrial nucleus at that time was reduced by half as compared with that at the early starved plasmodium stage. Dumbbell-shaped mitochondria appeared during the resting stage; these had two mt-nuclei, each located discretely at either side of the dumbbellshaped mitochondrion. Mitochondria containing 3-6 nuclei were also observed, at low frequency; multinucleated mitochondria persisted up to meiotic prophase I. The mitochondria in amoebae which originated from spores contained only one mt-nucleus. These results indicate that mitochondrial nuclear fusion occurs at some stage between spore germination and the amoeba stage, following mitochondrial fusion at the resting stage of sporulation in Physarum polycephalum.

\section{Acknowledgement}

This work was supported in part by Grants No. 511212 (T.K.) and 521708 (T.K.) from the Ministry of Education, Science and Culture of Japan.

\section{References}

Aldrich, H. C. 1967. The ultrastructure of meiosis in three species of Physarum. Mycologia 59: 127-148. Arescaldino, I. 1971. Evolution de la teneur en ADN des noyaux de Physarum polycephalum (Myxomycètes) au cours de la sporulation. C. R. Acad. Sci. D 273: 398-401.

D'Amato, F. 1977. Cytogenetics of differentiation in tissue and cell cultures. In: Applied and Fundamental Aspects of Plant Cell Tissue and Organ Culture (eds. Reinert, J. and Bajaj, Y. P. S.), pp. 343-357. Berlin-Heidelberg-New York: Springer.

Daniel, J. W. and Jarlfors, U. 1972. Light-induced changes in the ultrastructure of a plasmodial myxomycete. Tissue and Cell $4: 405-426$.

Guttes, E., Guttes, S. and Devi, R. V. 1967. Occurrence and morphology of a fibrous body in the mitochondria of the slime mold Physarum polycephalum. Experimentia 23: 713-715.

- , - and Rusch, H. P. 1961, Morphological observations on growth and differentiation of Physarum polycephalum grown in pure culture. Dev. Biol. 3: 588-614.

Hoffman, H. P. and Avers, C. J. 1973. Mitochondria of yeast: ultrastructural evidence for one giant, branched organelle per cell. Science 181: 749-751.

Kamiya, N. 1940. The control of protoplasmic streaming. Science 92: 462-463.

- and Seifriz, W. 1954. Torsion in a protoplasmic thread. Exp. Cell Res. 6: 1-16.

Kawano, S., Nishibayashi, S., Shiraishi, N., Miyahara, M. and Kuroiwa, T. 1983. Variance of ploidy in mitochondrial nucleus during spherulation in Physarum polycephalum. Exp. Cell Res. 149: 359-373.

-, Suzuki, S. and Kuroiwa, T. 1982. Structural homogeneity of mitochondrial DNA in the mitochondrial nucleoid of Physarum polycephalum. Biochim. Biophys. Acta 696: 290-298.

Kuroiwa, T. 1973. Studies on mitochondrial structure and function in Physarum polycephalum 1. Fine structure, cytochemistry and ${ }^{3} \mathrm{H}$-uridine autoradiography of a central body in mitochondria. Exp. Cell Res. 78: $351-359$.

-, Kawano, S. and Hizume, M. 1977. Studies on mitochondrial structure and function in Physarum polycephalum V. Behavior of mitochondrial nucleoids throughout mitochondrial division cycle. J. Cell 
Biol. 72: 687-694.

-, -, and - 1978. Studies on mitochondrial structure and function in Physarum polycephalum IV. Mitochondrial division cycle. Cytologia 43: 119-136.

-, Kitane, H., Watanabe, T. and Kawano, S. 1976. The general occurrence of mitochondrial nuclei in the true slime mold. J. Electron Micros. 25: 103-105.

Miyakawa, I., Aoi, H., Sando, N. and Kuroiwa, T. 1984. Fluorescence microscopic studies of mitochondrial nucleoids during meiosis and sporulation in the yeast, Saccharomyces cerevisiae. J. Cell Sci. 66: 2138.

Mohberg, J., Babcock, K. L., Haugli, F. B. and Rusch, H. P. 1973. Nuclear DNA content and chromosome numbers in the myxomycete Physarum polycephalum. Dev. Biol. 34: 228-245.

- and Rusch, H. P. 1971. Isolation and DNA content of nuclei of Physarum polycephalum. Exp. Cell Res. 66: 305-316.

Nagl, W. 1978. Endopolyploidy and Polyteny in Differentiation and Evolution. Amsterdam-New YorkOxford: Elservier/North-Holland Biomedical Press.

Nicholls, T. J. 1972. The effects of starvation and light on intra-mitochondrial granules in Physarum polycephalum. J. Cell Sci. 10: 1-14.

Nishibayashi, S. and Kuroiwa, T. 1982. Behavior of leucoplast nucleoids in the epidermal cell of onion (Allium cepa) bulb. Protoplasma 110: 177-184.

- and - 1985. Division of mitochondrial nuclei in protozoa, a green alga and a higher plant. Cytologia 50: $75-82$.

Sando, N., Mıyakawa, I., Nishibayashi, S. and Kuroiwa, T. 1981. Arrangement of mitochondrial nucleoids during life cycle of Saccharomyces cerevisiae. J. Gen. Appl. Microbiol. 27: 511-516.

Sauer, H. W., Babcock, K. L. and Rusch, H. P. 1969. Sporulation in Physarum polycephalum. A model system for studies on differentiation. Exp. Cell Res. 57: 319-327.

Spurr, A. R. 1969. A low viscosity resin embedding medium for electron microscopy. J. Ultrastruct. Res. 26: 31-43.

Stevens, B. 1981. Mitochondrial structure. In the Molecular Biology of the Yeast Saccharomyces cerevisiae. Life Cycle and Inheritance. (eds. Strathern, J. N., Jones, E. W. and Broach, J. R.) pp. 471-504. Cold Spring Harbor Laboratory.

Yoshimoto, Y. and Kamiya, N. 1978. Studies on contraction rhythm of the plasmodial strand 1. Synchroni zation of local rhythms. Protoplasma 95: 89-99. 